\title{
Solubilization of Carbon Nanotubes Using Microplasma Generated in Water
}

\author{
Junya Suehiro and Kiminobu Imasaka \\ Kyushu University, Motooka 744, Nishi-ku, Fukuoka, 819-0395 Japan \\ Fax:+81-92-802-3683, e-mail: suehiro@ees.kyushu-u.ac.jp
}

The present study demonstrates a novel preparation method for water-soluble carbon nanotubes (CNTs) using microplasma generated in water suspension of CNTs. The microplasma was achieved by generating pulsed streamer discharges, which were triggered by applying multiple square high voltage pulses to a wire-to-plane electrode system immersed in the CNT suspension. The CNTs treated by the microplasma were solubilized and homogeneously dispersed in water for a month or longer. The functional group of $-\mathrm{OH}$ seemed to be bound to the CNT surface and responsible for the solubilization effect. The -OH group might be formed by chemical reactions between $\mathrm{O}^{\dagger}$ and $\mathrm{H}^{\dagger}$ radicals, which were detected by optical emission spectroscopy of the microplasma. The proposed method does not need any particular chemical agents or additives for solubilization. Of particular importance is its simplicity and time-efficiency in contrast to the usual time-consuming chemical treatments developed thus far. It was demonstrated that bio-nano interfaces between the water-soluble CNTs and biological cells could be readily fabricated by simultaneous electrokinetic manipulation of them in water using dielectrophoresis (DEP). The DEP trapped CNTs could serve as a $\mathrm{NO}_{2}$ gas sensor, showing that the surface adsorption of gas molecules on the CNTs did not deteriorate by the microplasma treatment.

Key words: Carbon nanotube, Microplasma, Solubilization, Bio-Nano interface, Dielectrophoresis

\section{INTRODUCTION}

Carbon nanotubes (CNTs) are widely used in the variety fields because of the unique electrical, mechanical and chemical properties. However, poor solubility of CNTs in water or organic solvents limits use of the CNTs for many potential applications. Thus far, several approaches have been attempted to solubilize CNTs, such as covalent or noncovalent functionalization $[1,2]$, chemical oxidation [3], addition of surfactants [4], polymer wrapping [5] and use of DNA (deoxyribonucleic acid) as a solubilizer [6]. Although these methods were successful, they need some particular chemical agents or additives for solubilization. The present study demonstrated a novel preparation method for water-soluble CNTs using microplasma generated in water suspension of CNTs [7]. The microplasma was achieved by generating pulsed streamer discharges, which were triggered by applying multiple square high voltage pulses to a wire-to-plane electrode system immersed in the CNT suspension. Thus far, a pulsed streamer discharge in water has been applied mainly to water purification processes such as removal of organic pollutants and sterilization of bacteria [8]. To the best of our knowledge, modification of nanomaterials by a pulsed streamer discharge in water has not been investigated or reported yet, while there are some reports regarding the surface modification of CNTs using plasma treatments such as a dielectric barrier discharge and a radio frequency glow discharge in gases $[9,10]$. The proposed method does not need any particular chemical agents or additives, and has higher simplicity and time-efficiency than conventional chemical treatments. The solubilization efficiency was considerably improved by introducing oxygen gas bubbles into the CNT suspension. It was also demonstrated that bio-nano interfaces could be readily fabricated by simultaneous manipulation of the water-soluble CNTs and biological cells in water using dielectrophoresis.

\section{EXPERIMENTAL DETAILS}

Fig.1 shows a schematic of the experimental setup for the CNT solubilization using the pulsed streamer discharge (microplasma) in water with oxygen gas bubbling. Single-walled CNTs (SWCNTs, purchased from Sigma Aldrich Inc., U.S.A) and multi-walled CNTs (MWCNTs, purchased from Shenzhen Nanotech Port Co., Ltd., China) were used as samples to be

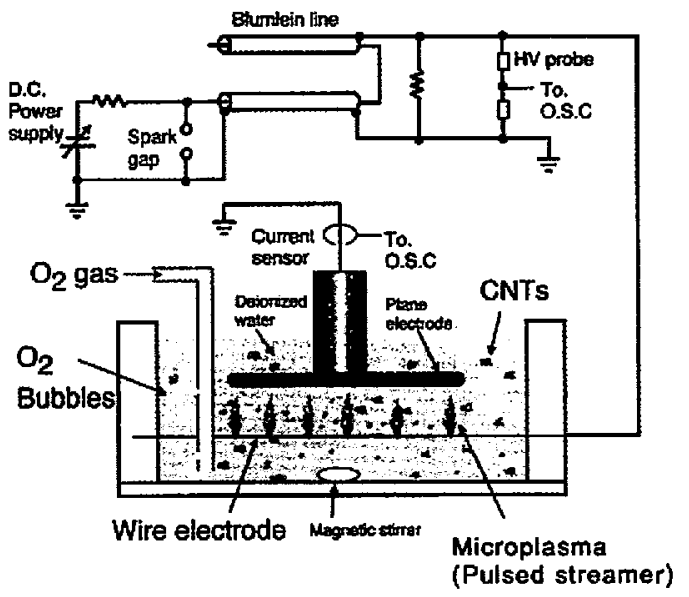

Fig.1 Experimental setup. 
solubilized by the proposed method. The SWCNT had $15 \mathrm{~nm}$ diameter as a bundle, $2-5 \mu \mathrm{m}$ lengths and $50-70 \%$ purity. The MWCNT had $60-100 \mathrm{~nm}$ diameter, $5-15 \mu \mathrm{m}$ length and $95 \%$ purity. The CNTs were suspended in deionized water without any mechanical mixing such as an ultrasonication $(100 \mu \mathrm{g} / \mathrm{ml}$ concentration). The CNTs suspensions $(60 \mathrm{ml})$ were filled in a rectangular acrylic chamber $(60 \mathrm{~mm} \times 30 \mathrm{~mm}$ $x 30 \mathrm{~mm}$ ) in which a wire-to-plane electrode system was installed. A tungsten wire $(60 \mu \mathrm{m}$ diameter, $60 \mathrm{~mm}$ length) was used as a high voltage electrode. A stainless steel plane electrode ( $28 \mathrm{~mm} \times 58 \mathrm{~mm}$ ) was connected to the ground. The gap length between the wire and the plane electrode was $13 \mathrm{~mm}$. The pulsed high voltage was generated by using a homemade pulsed power generator based on a Blumlein transmission line. The pulsed power generator could generate a square voltage waveform with the maximum amplitude of $35-40 \mathrm{kV}$ at a repetition rate of $15 \mathrm{~Hz}$. The CNT suspension was continuously stirred using a magnetic stirrer to inhibit precipitation of CNTs. Oxygen gas bubbles were introduced into in the suspensions through a Teflon tube at a flow rate of $100 \mathrm{ml} / \mathrm{min}$.

\section{RESULTS}

3.1 Microplasma generated by pulsed streamer discharge Fig.2 shows a typical pulsed voltage waveform generated by the pulsed power generator as well as a current waveform observed when the pulsed high voltage was applied to the wire electrode immersed in the MWCNT suspension. The positive polarity voltage was used because a more stable streamer discharge was obtained than using negative polarity voltage. The rise time and the duration (full wave at half maximum, FWHM) of the pulse were approximately $50 \mathrm{~ns}$ and $330 \mathrm{~ns}$, respectively. The pulsed current had the almost same duration as the applied voltage. At the voltage front, the current oscillated probably due to the inductance of the discharge circuit. After the oscillation, the current once reached a constant value about $10 \mathrm{~A}$, and began to increase again in the latter half period. As shown in Fig.3, filamentary streamer discharges were generated in the electrode gap along the wire electrode when the pulsed high voltage was applied to the wire electrode. The streamer filament was composed of microplasma channels triggered by electron avalanches propagating from the wire surface where a high electric field appeared. The maximum electric field strength on the wire surface could become as high as $1 \mathrm{MV} / \mathrm{mm}$ with a pulsed voitage amplitude of $40 \mathrm{kV}$. A time-resolved optical observation using a photodiode (Fig.2c) revealed that the streamer generation was synchronized with the current increase in the latter half period. These results suggest that the latter current increase can be regarded as the conductive current generated by the streamer discharge ionization, which is imposed on the conductive current flowing through MWCNT suspension. The peak value of the streamer current component was estimated in the range from $5 \mathrm{~A}$ to $20 \mathrm{~A}$ depending on the voltage amplitude. The streamer current could be also controlled by the voltage pulse width, which proportionally increased with the length of the Blumlein transmission line.

\subsection{Solubilization of SWCNTs}

Before the microplasma treatment, the SWCNT suspension was almost optically transparent because many aggregations of insoluble SWCNTs were formed and precipitated as shown Fig.4a. After the microplasma treatment for 10 min without oxygen gas bubbling, the CNT suspension gradually turned into a light gray color indicating that the CNTs were partially solubilized and well dispersed in water (Fig.4b). The solubility of SWCNTs was poorer than that of MWCNTs treated under an identical condition [7]. By introducing oxygen

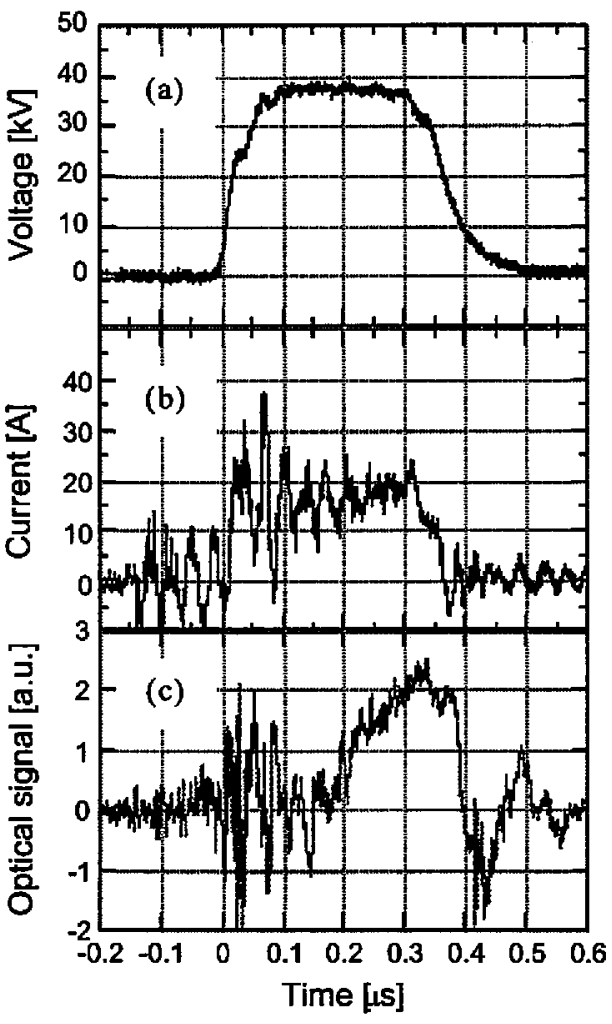

Fig.2 Example of typical waveforms. (a) Voltage. (b) Current. (c) Light emission.

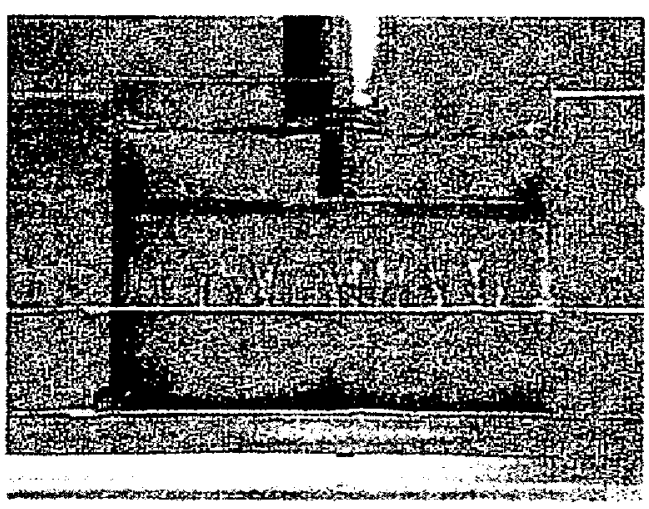

Fig.3 Photo of pulsed streamer discharge (microplasma) generated between wire-to-plane electrodes immersed in water suspension of CNTs. 

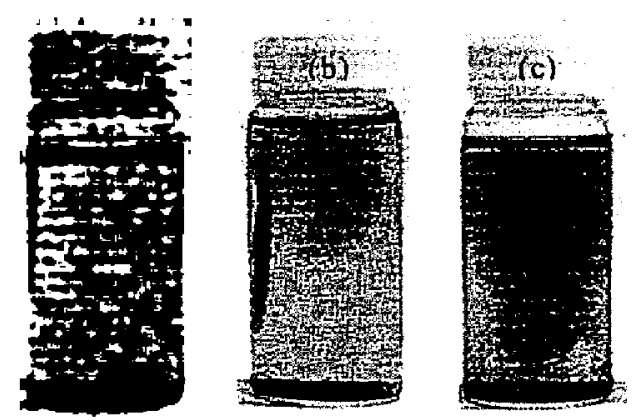

Fig.4 Photographs of SWCNTs suspended in water. (a) Before microplasma treatment. (b) After microplasma treatment without $\mathrm{O}_{2}$ bubbling. (c) After microplasma treatment with $\mathrm{O}_{2}$ bubbling.

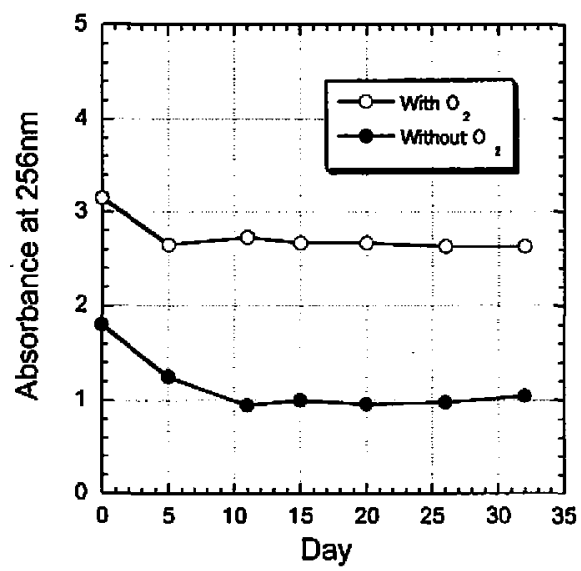

Fig.5 Temporal change of absorbance of SWCNT suspension at $256 \mathrm{~nm}$ after microplasma treatment with or without $\mathrm{O}_{2}$ bubbling.

gas bubbles into the suspension during the microplasma treatment, however, the solubility of SWCNTs was considerably enhanced (Fig.4c). Effects of the oxygen gas bubbling can be confirmed in Fig.5, which depicts temporal change of UV-Vis absorbance peak of the SWCNT suspension after the treatment. The absorbance at $256 \mathrm{~nm}$, which can be a measure of solubility of the SWCNT in water [11], was increased by 2.5 times by combining oxygen bubbling with the microplasma treatment. It was confirmed that the oxygen bubbling can increase solubility of the SWCNT, which could not solubilized only by the microplasma treatment even with longer processing time.

Fig.6 shows the SWCNT size distribution in water before and after the microplasma treatment with oxygen bubbling, which was measured by using a laser diffraction particle size analyzer (SALD-7100, Shimadzu, Japan) after sample storage for a month. After the treatment, the SWCNT size was drastically reduced by 2-3 orders of magnitude. The mean size was about $100 \mathrm{~nm}$, which is larger than diameter of the SWCNT bundle $(15 \mathrm{~nm})$. However, considering high aspect ration of the SWCNT, the result may imply that the SWCNT bundles are well dispersed in water without forming their aggregations.

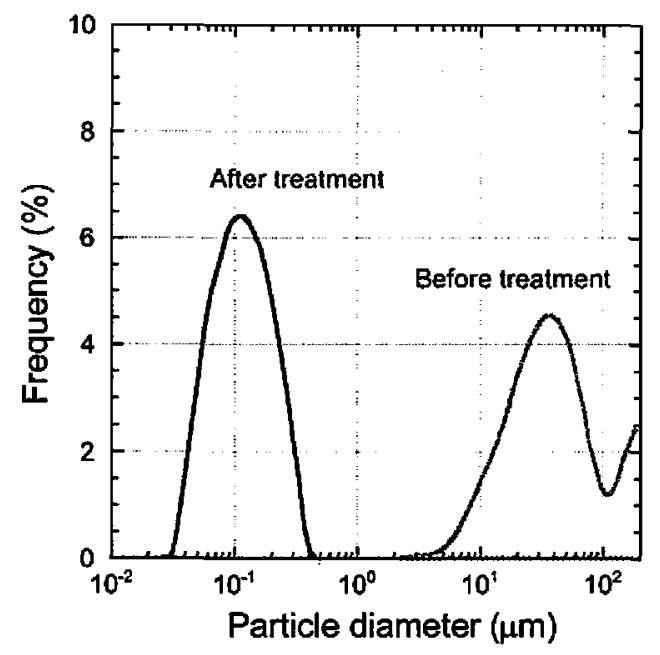

Fig.6 The SWCNT size distribution in water before and after the microplasma treatment with oxygen bubbling.

\section{DISCUSSION}

4.1 Optical emission spectra from microplasma in water

In the previous study [7], Fourier transformation infrared (FTIR) spectroscopy was used to analyze surface modification of MWCNTs. It was found that the hydroxyl $(-\mathrm{OH})$ groups, which are known to impart a hydrophilic nature to carbon material, were introduced on the MWCNTs surface. Optical emission spectra from the microplasma generated in the MWCNT suspension showed that highly oxidative $\mathrm{O}^{\dagger}$ and $\mathrm{H}^{-1}$ radicals were generated in water. These results suggest that the functionalization of the MWCNT surface by $-\mathrm{OH}$ group can be attributed to the $\mathrm{O}^{\dagger}$ and $\mathrm{H}^{\dagger}$ radicals.

The FTIR analysis of the microplasma-treated SWCNTs is now being conducted and the details have not been clarified yet. However, it seems that a similar mechanism lies behind the solubilization process of the SWCNTs in water using microplasma. It has been reported that the pulsed streamer discharge generates various active chemical species such as $\mathrm{OH}^{\dagger}, \mathrm{O}^{\dagger}, \mathrm{H}^{\dagger}$, $\mathrm{H}_{2} \mathrm{O}_{2}, \mathrm{HO}_{2}$ and $\mathrm{O}_{3}$ in water [12]. These radicals might introduce $-\mathrm{OH}$ groups on the CNT surface as a result of chemical reactions. Optical emission spectra of the pulsed streamer discharge plasma generated in the SWCNT suspension were measured using a multichannel spectrometer. As depicted in Fig.7, three distinctive peaks were observed along with a strong continuum in the wavelength range of visible light. The emission lines at $486 \mathrm{~nm}$ and $656 \mathrm{~nm}$ are attributed to excited hydrogen atoms $H_{\beta}$ and $H_{\alpha}$, respectively $[12,13]$. The peak at $777 \mathrm{~nm}$ is assigned to the emission line of atomic oxygen in an excited state $[12,13]$. Although $\mathrm{H}^{\dagger}$ and $\mathrm{O}^{+}$radicals were optically detected, the $\mathrm{OH}^{-1}$ radical, which seemed to be most responsible for the introduction of $-\mathrm{OH}$ groups and has been observed in similar experiments performed by other researchers [ 12 , 13], was not observed. However, it is known that $-\mathrm{OH}$ groups can be formed on the surface or open ends of CNTs during the oxidation process in oxygen or air [14]. 


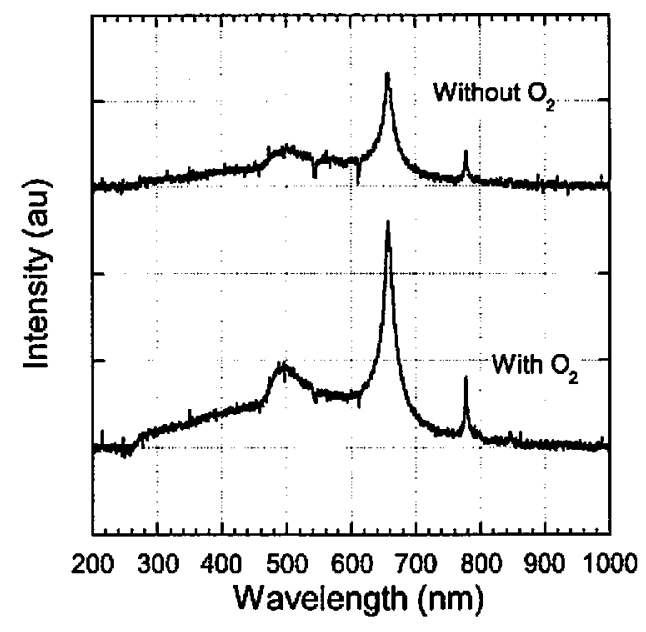

Fig.7 Optical emission spectra from the microplasma generated in the SWCNT suspension with or without $\mathrm{O}_{2}$ bubbling.

The oxidative $\mathrm{O}^{-1}$ radicals can react with the SWCNT surface first, and then may form $-\mathrm{OH}$ groups as a result of chemical reaction with $\mathrm{H}^{\dagger}$ radicals. The shock wave induced by the pulsed streamer may enhance the radical reactions since it can mechanically break the CNT aggregations into smaller particles and increase the surface area.

Fig.7 also shows that the emission line peaks became larger indicating that more radicals were generated by injecting oxygen gas bubbles into the SWCNT suspension water. There might be two possible reasons for the enhanced radical formation. Firstly, $\mathrm{O}^{\dagger}$ radical can be increased because more oxygen atoms are available. However, this cannot explain why $\mathrm{H}^{*}$ radicals also increase. The second possible mechanism is that the electrons in the gaseous phase can be energized to obtain higher kinetic energy than in liquid phase due to the longer mean free path. This idea predicts that the similar effect can be achieved using other gases than oxygen. Actually, additional experiments confirmed that the SWCNT solubilization and the radical emissions were enhanced using argon gas bubbles. The detail mechanism is now under investigation.

4.2 Simultaneous manipulation of the water-soluble CNTs and biological cells using dielectrophoresis

The authors have developed an electrokinetic manipulation technique for biological cells [15] and CNTs [16] using positive dielectrophoresis (DEP). DEP is the electrokinetic motion of dielectrically polarized materials in non-uniform electric fields and is currently an active area of research for manipulation of microscale biological materijals as well as nanomaterials.

For the manipulation, cells and nanotubes need to be uniformly suspended in a liquid. Biological cells are generally suspended in a water-based medium, while CNTs are ultrasonicated in an organic solution such as ethanol because of their poor solubility in water. Since biological cells cannot stay alive in ethanol, cells and CNTs cannot be simultaneously manipulated in the same suspension. The water-soluble CNTs prepared by the proposed technique overcome this technological barrier and might realize a one-step assembly of a nano/micro system composed of both biological and non-biological building blocks. Fig. 8 shows a scanning electron microscopy (SEM) image of MWCNTs and Escherichia coli $(E$. coli) bacteria, which were trapped onto a microelectrode under action of the positive DEP [17]. The water soluble MWCNTs, which were prepared by the microplasma treatment, were suspended in water with $E$. coli cells. An interdigitated microelectrode of thin chrome film ( $100 \mathrm{~nm}$ thickness) was patterned on a glass substrate by the photolithography technique. The electrode had a castle-wall pattern in order to form high and low electric field regions periodically. Each electrode finger had a $5 \mathrm{~mm}$ length and $5 \mu \mathrm{m}$ minimum clearance. The castellations were squares with sides of $50 \mu \mathrm{m}$. The castle wall electrode was surrounded by a silicon rubber spacer to form a sealed chamber in which the suspension was stored. The suspension was continuously fed into the microelectrode chamber from a reservoir by a peristaltic pump. The DEP trapping of MWCNTs and bacteria on the microelectrode was performed with an ac voltage of $100 \mathrm{kHz}$ frequency and $10 \mathrm{~V}$ amplitude (peak-to-peak value).

It seemed some bacteria were preferably trapped on the CNT surface, where higher electric field and stronger DEP force might be generated due to high aspect ratio of CNT. As a result, bio-nano interfaces between $E$, coli cells and CNTs were automatically formed.

Zhou et al. conducted similar experiments and found that bacteria trapping was enhanced by mixing water soluble SWCNTs in the bacteria suspension [18]. They suggested that the enhanced DEP trap of bacteria was probably due to higher electric field and resultant stronger DEP force generated near CNT. Since the MWCNTs used in the present study have larger diameter than SWCNTs, it is expected that DEP force enhancement may become lower. Effects of CNT size should be investigated in detail later on. Besides the enhancement of DEP trap of bacteria, simultaneous DEP manipulation of bacteria and water-soluble CNTs has some advantages and potential applications. Fig.9 illustrates one possible application of bacteria/CNT interfaces, which can be readily and easily assembled by the proposed manipulation technique. It has been

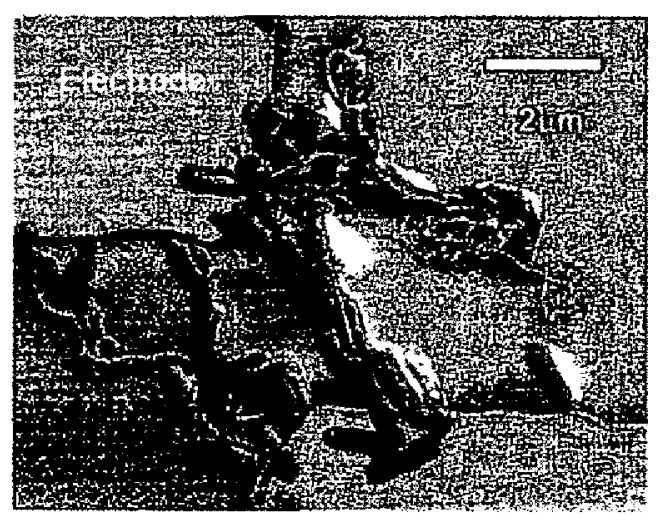

Fig.8 SEM images of MWCNTs and $E$. coli cells which were simultaneously suspended in water and trapped onto the microelectrode under action of positive DEP. 

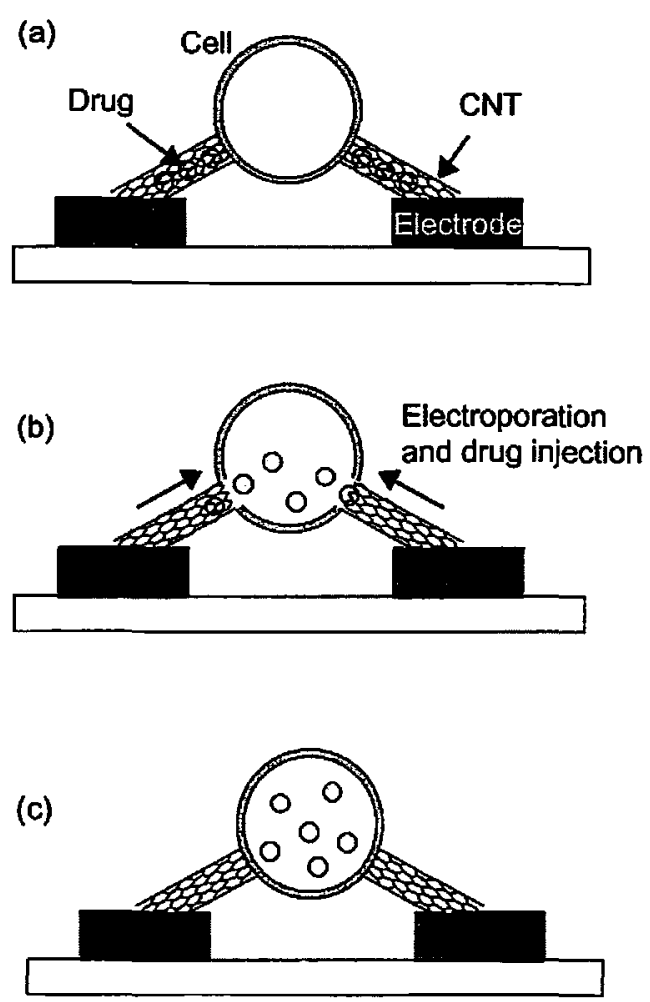

Fig.9 The principle of the drug delivery into a biological cell using bio-nano interfaces. (a) The drug-loaded CNTs and biological cell are simultaneously trapped in the microelectrode gap by positive DEP. (b) Injection of drugs into the cell through electroporated cell membrane. (c) Drug delivery process is completed with high efficiency and selectivity.

demonstrated that CNTs can be loaded with various chemicals or drugs [19]. By simultaneous DEP manipulation of the drug-loaded CNTs and biological cells in water, one can make CNTs contact with cells. After that, electroporation is induced by applying high electric field pulses so that drugs loaded in CNTs can be transferred into the cell interior through electroporated cell membrane. It is expected that electroporation efficiency can be improved by high electric field formed around the surface or tip of CNTs. The proposed protocol may realize a drug delivery systems (DDS) with high efficiency and selectivity.

4.3 Effects of the microplasma treatment on DEP fabricated CNT gas sensor

It is known that CNTs can be solubilized in water by using Sodium Dodecylsulfate (SDS) surfactant [4]. Since the SDS molecules wrap around a CNT, it is expected that the SDS wrapping can affect gas molecule adsorption on the CNTs after drying out. Using DEP manipulation technique mentioned above, two CNT gas sensors were fabricated as shown in Fig.10. One is made from the microplasma treated SWCNTs, and the other is made from SDS wrapped ones. The SEM observation revealed that both kinds of water-solubilized CNTs were similarly trapped onto the microelectrode by positive DEP. After the DEP trap, the water was dried out to achieve SWCNTs which serve as a gas sensor [16]. As shown in Fig.1l, the SDS wrapped CNTs did not respond to $1 \mathrm{ppm} \mathrm{NO}_{2}$ gas at room temperature. On the other hand, the microplasma treated CNTs responded to $\mathrm{NO}_{2}$ gas. The sensor response was compatible to that obtained with CNT gas sensor fabricated by DEP manipulation of CNTs ultrasonicated in ethanol [16]. This result suggests that the SDS molecules, which wrap around CNTs in water and may be retained after drying out, prevents $\mathrm{NO}_{2}$ molecules from being adsorbed to the CNT surface. Contrary to this, the functionalization groups introduced by the microplasma treatment seem to

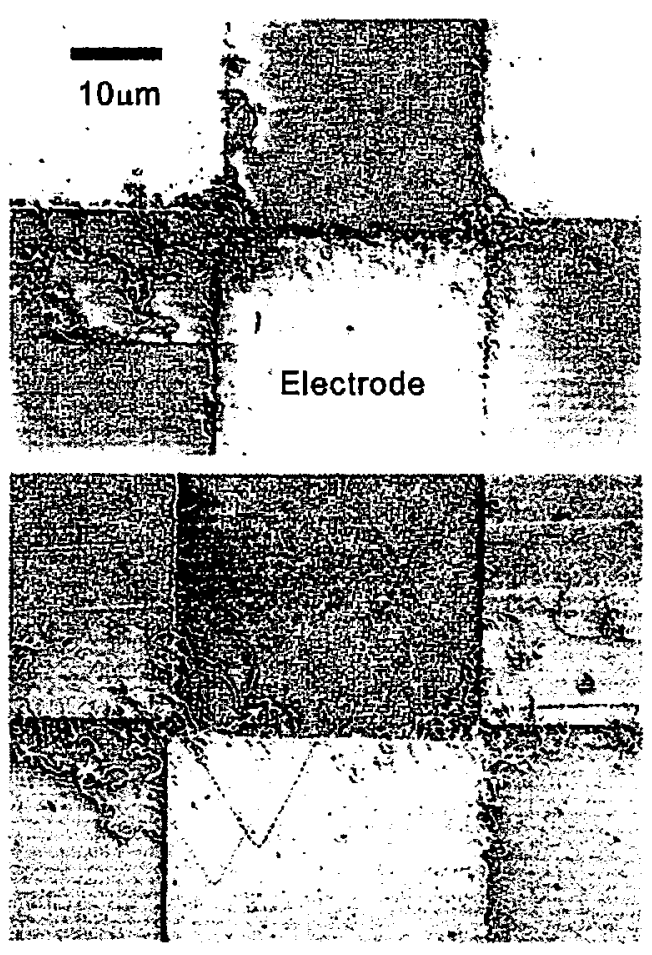

Fig.10 SEM images of MWCNTs trapped onto the microelectrode by positive DEP to fabricate a CNT gas sensor. (a) MWCNTs solubilized by SDS. (b) MWCNTs solubilized by microplasma.

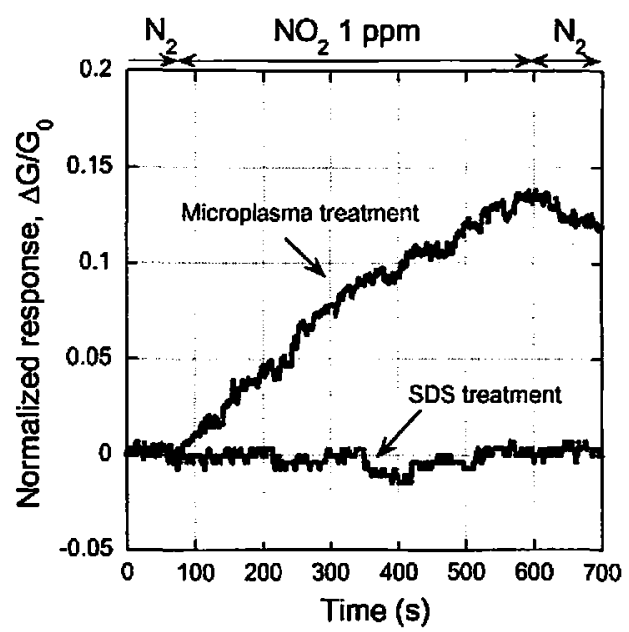

Fig.11 $\mathrm{NO}_{2}$ responses of the MWCNT gas sensors. 
have less influence on the CNT surface adsorption. This can be a great advantage when the microplasma treated CNTs are used for sensor and transducer applications.

\section{CONCLUSION}

Water-soluble SWCNTs were obtained by using a microplasma generated by pulsed streamer discharge developed in water suspension of CNTs in water. The repetitive pulsed streamer discharge was generated by applying multiple square voltage pulses to a wire-to-plane electrode system immersed in the SWCNT suspension. The SWCNTs treated by the pulsed streamer discharge were solubilized and were homogeneously dispersed in water for a long time. The functional group of $-\mathrm{OH}$ bound to the CNT surface seemed to be responsible for the solubilization effect. The $-\mathrm{OH}$ group might be formed by chemical reactions between $\mathrm{O}^{\dagger}$ and $\mathrm{H}^{-1}$ radicals, which were detected by optical emission spectroscopy. By injecting gas bubbles into the SWCNT suspension during the microplasma treatment, the solubilization efficiency was improved. Potential applications of the solubilized CNTs were demonstrated in construction of bio-nano interfaces and gas sensors using DEP manipulation technique.

\section{Acknowledgments}

This work was partly supported by Grants-in-Aid for Scientific Research from the Japan Society for the Promotion of Science (No. 18360200).

\section{References}

[1] J. H. T. Luong, S. Hrapovic, D. Wang, F. Bensebaa and B. Simard, Electroanalysis, 16, 132-9 (2004).

[2] N. Nakashima, Y. Tomonari and H. Murakami, Chem. Lett., 31, 638-9 (2002).
[3] T. Nakajima and Y. Matsuo, Carbon, 32, 469-75 (1994).

[4] M. F. Islam, E. Rojas, D. M .Bergey, A. T. Johnson and A. G Yodh, Nano Lett., 3, $269-73$ (2003).

[5] J. Chen, H. Liu, W. A. Weimer, M. D. Halls, D. H. Waldech and G. C. Walker, J. Am. Chem. Soc., 124, 9034-5 (2002).

[6] M. Zheng, A. Jagota, E. D. Semke, B. A. Diner, R. S. Mclean, S. R. Lustig, R. E. Richardson and N. G Tassi, Nature Materials, 2, 338-42 (2003).

[7] K. Imasaka, J. Suehiro, Y. Kanatake, Y. Kato and M. Hara, Nanotechnology, 17, 3421-7 (2006).

[8] H. Akiyama, IEEE Trans. Dielectr. Electr. Insul., 7, 646-53 (2000).

[9] T. I. T. Okpalugo, P. Papakonstantinou, H. Murphy, J. Mclaughlin and N. M. D. Brown, Carbon, 43, $2951-9$ (2005).

[10]Q. Chen, L. Dai, M. Gao, S. Huang and A. Mau, J. Phys. Chem. B, 105, 618-22 (2001).

[11] K. Imasaka, Y. Kato, J. Suehiro and N. Sano, Proc. of 2006 Annual Conference of Fundamentals and Materials Society IEE Japan, 6-11 (2006).

[12]B. Sun, M. Sato and J. S. Clements, J. Electrost, 39, 189-202 (1997).

[13]N. J. Salazar, N. Bonifaci, A. Denat and O. Lesaint, Proc. of the IEEE Int. Conf. on Dielectric Liquids, $91-4(2005)$.

[14]X. L. Xie, Y. W. Mai and X. P. Zhou, Mater. Sci. Eng. R, 49, 89-112 (2005).

[15]J. Suehiro, R Hamada, D. Noutomi, M. Shutou and M. Hara, J. Electrost., 57. 157-68 (2003).

[16]J. Suehiro, G Zhou, H. Imakiire , W. Ding and M. Hara, Sensors Actuators B 108, 398-403 (2005).

[17] A. Ohtsubo, J. Suehiro and K. Imasaka, Proc. of the 23rd Sensor Sympositum on Sensors, Micromachines and Applied Systems, 366-9 (2006).

[18]R. Zhou, P. Wang and H. Chang, Electrophoresis, 27, 1376-85 (2006).

[19]A. Bianco, K. Kostarelos and M. Prato, Current Opinion in Chemical Biology, 9, 674-9 (2005).

(Received December 9, 2006;Accepted February 2, 2007) 